\title{
On retractability and its relationships with lattices associated to a ring
}

\author{
Rodrigo Domínguez-López (D), Oscar Alberto Garrido-Jiménez (D), Hugo Alberto \\ Rincón-Mejía* (D), Manuel Gerardo Zorrilla-Noriega (D) \\ Facultad de Ciencias, Universidad Nacional Autónoma de México Circuito Exterior, C.U., C.P. 04510, \\ Ciudad de México, México
}

\begin{abstract}
The purpose of this work is threefold. First, we explore some relationships between retractability and some lattices of classes of modules. Secondly, we weaken the hypothesis of a result of Ohtake characterizing rings over which all radicals are left exact. In the last section of this work, we introduce a binary relation between modules that produce a Galois connection between the lattice of natural classes and the lattice of conatural classes, and we obtain some results about it.
\end{abstract}

Mathematics Subject Classification (2020). 16D80, 16S90, 16S99

Keywords. left mod-retractable ring, parainjective module, paraprojective module, left semiartinian ring, left max ring, left local ring, left duo ring, left V-ring, strongly left mod-retractable ring

\section{Introduction}

In this work $R$ denotes an associative ring with 1 and $R$-Mod denotes the category of left unitary $R$-modules. Unless otherwise stated, all modules are left $R$-modules and all classes are closed under taking isomorphic copies of their members. Depending on the context, "quotient" may refer to an actual quotient or to a homomorphic image.

We say that a module $M$ is retractable if $\operatorname{Hom}_{R}(M, N) \neq 0$ for each nonzero submodule $N$ of $M$. When all left $R$-modules are retractable, we say that $R$ is a left mod-retractable ring. It is known that $R$ is a left mod-retractable ring if and only if each torsion class is hereditary.

The main subject of this paper is retractability and its relationships with some lattices associated to a ring. Considered here are the big lattice of torsion theories, the lattice of hereditary torsion theories, the lattices of natural classes and of conatural classes, and the big lattice of preradicals.

Natural classes were introduced and studied by Dauns and Zhou (see [8]). These are classes of modules closed under taking submodules, direct sums and injective hulls. A natural class can be seen also as a pseudocomplement in the big lattice of hereditary classes.

\footnotetext{
*Corresponding Author.

Email addresses: cucumeto@ciencias.unam.mx (R. Domínguez-López), oagj@ciencias.unam.mx (O.A. Garrido-Jiménez), hurincon@gmail.com (H.A. Rincón-Mejía), mgzn@hotmail.com (M.G. Zorrilla-Noriega) Received: 25.09.2020; Accepted: 24.02.2021
} 
This situation can be dualized, producing conatural classes, defined as the pseudocomplements in the big lattice of cohereditary classes. ${ }^{\dagger}$ Like natural classes, conatural classes conform a Boolean lattice.

In [2], the concepts of a simple parainjective module (that is, if $S$ is a simple submodule of some module $M$, then $S$ is a quotient of $M$ ) and of a simple paraprojective module (that is, if $S$ is a simple quotient of some module $M$, then $S$ embeds in $M$ ) were introduced. It is easy to see that if a ring is mod-retractable then every simple module is parainjective. If in addition, a ring fulfills that every simple module is paraprojective, then the converse holds (as will be shown in this paper).

We will show that if every simple module is parainjective, then the ring is left max (that is, every proper submodule is contained in a maximal submodule) and that if every simple module is paraprojective, then the ring is left semiartinian.

In [3] it is shown that every conatural class is a hereditary torsion class if and only if every simple module is parainjective and that every natural class is cohereditary if and only if every simple module is paraprojective.

In [12] Ohtake's Theorem states that, for a commutative ring $R, R$ being semiartinian and regular is equivalent to all radicals over $R$ being left exact. In the second part of this work we extend this result for left duo rings.

We say that a module $M$ is strongly retractable if $\operatorname{Hom}_{R}\left(N^{\prime}, M^{\prime}\right) \neq 0$ for each nonzero quotient $N^{\prime}$ of $M$ and each nonzero submodule $M^{\prime}$ of $M$. We call a ring strongly left modretractable if each left $R$-module is strongly retractable. In the third part of this work, we characterize this kind of rings. In the development process of this characterization, we obtain a Galois connection between the lattice of natural classes and the lattice of conatural classes.

\section{Retractability and some lattices of module classes}

Definition 2.1. A module $M$ is retractable if $\operatorname{Hom}(M, N) \neq 0$ for each nonzero $N \leq M$. A ring $R$ is left mod-retractable (or simply mod-retractable, if there is no risk of ambiguity) if each $R$-module is retractable.

Definition 2.2. A module $M$ is a parainjective module if for each module $N$, whenever there is some monomorphism $f \in \operatorname{Hom}(M, N)$ there exists an epimorphism $g \in$ $\operatorname{Hom}(N, M)$. A module $M$ is a paraprojective module if for each $N$, whenever there is some epimorphism $f \in \operatorname{Hom}(N, M)$ there exists a monomorphism $g \in \operatorname{Hom}(M, N)$.

Recall that a module is said to be semiartinian if each of its nonzero quotients contains a simple submodule. A module $M$ is coatomic if each of its proper submodules is contained in a maximal submodule of $M$. A ring $R$ is left semiartinian if every $R$-module is semiartinian. A ring $R$ is left max if every $R$-module is coatomic.

It is easy to see that a ring $R$ is left semiartinian if and only if ${ }_{R} R$ is a semiartinian module. Also, $R$ is left semiartinian if and only if each nonzero $R$-module contains a simple submodule. Correspondingly, $R$ is left max if and only if each nonzero $R$-module has a simple quotient.

Remark 2.3. The following statements hold.

(1) If each simple module is paraprojective, then the ring is a left semiartinian ring.

(2) If each simple module is parainjective, then the ring is left max.

(3) Over any mod-retractable ring, each simple module is parainjective.

${ }^{\dagger} \mathrm{A}$ cohereditary class is a class of modules closed under taking quotients. 


\section{Proof.}

(1) If $M$ is a nonzero module, take a cyclic nonzero submodule of $M$, say $R x$. This cyclic module has a simple quotient $S$, which, being paraprojective, embeds in $R x$. Thus $M$ has a simple submodule.

(2) If $M$ is a nonzero module, take a nonzero cyclic submodule $R x$ and take a simple quotient $S$ of $R x$. Composing with the inclusion of $S$ in its injective hull $E(S)$, we have a nonzero homomorphism from $R x$ to $E(S)$, which extends to a nonzero homomorphism $f: M \rightarrow E(S)$. As $S$ is an essential submodule of its injective hull, we have that $S$ embeds in $f(M)$. As $S$ is parainjective, then $S$ is a quotient of $f(M)$ and thus also of $M$.

(Or, since $S$ is a quotient of a submodule of $M$, a push-out shows that $S$ embeds in a quotient of $M$, so, by parainjectivity, $S$ is a quotient of $M$.)

(3) If $S$ is a simple submodule of $M$, then there exists a nonzero homomorphism from $M$ to $S$, which is necessarily an epimorphism.

Recall that a left $V$-ring is a ring over which every simple module is injective.

Example 2.4. The following examples show that the converses of the statements in Remark 2.3 do not hold.

(1) Consider the ring $\left(\mathbb{Z}_{2}\right)^{\mathbb{N}}$ and let $R$ be the subring spanned by $\left(\mathbb{Z}_{2}\right)^{(\mathbb{N})}$ and 1 , that is, $R$ consists of all eventually constant sequences of 0 's and 1 's. We have that $R$ is a commutative ring and $\operatorname{soc}(R)=\left(\mathbb{Z}_{2}\right)^{(\mathbb{N})}$ is a maximal and essential ideal of $R$. Then, $S=R / \operatorname{soc}(R)$ is a simple singular $R$-module. Since all semisimple modules are semiartinian, and since the class of semiartinian modules is closed under extensions, the short exact sequence $0 \rightarrow \operatorname{soc}(R) \rightarrow R \rightarrow S \rightarrow 0$ shows that $R$ is semiartinian. Besides, $\operatorname{soc}(R)=\bigoplus_{i \in \mathbb{N}} R e_{i}$ where $e_{i} \in R$ has 1 at the $i$ th place and 0 elsewhere, each $R e_{i}$ being a simple direct summand of $R$, so that $\operatorname{soc}(R)$ is projective semisimple. We know that any one simple module is either projective or singular, so, $\operatorname{soc}(R)$ being essential in $R, R$ is necessarily a nonsingular ring. Therefore, $\operatorname{Hom}(S, R)=0$. It follows that $S$ is not a paraprojective module.

(2) Let $R$ be the ring of all matrices of the form $\left(\begin{array}{ll}a & b \\ 0 & c\end{array}\right)$, with $a, b, c \in \mathbb{Z}_{2}$. $R$ is a semiprimary ring, whence it is a left and right perfect ring. Then, being left perfect, $R$ is a left max ring. The matrices of the form $\left(\begin{array}{ll}a & 0 \\ 0 & 0\end{array}\right)$, with $a \in \mathbb{Z}_{2}$, are the elements of a simple projective module $S$ which is not injective (it is a direct summand of $R$ and it is essential in the left $R$-module $\left.\left\{\left(\begin{array}{cc}a & 0 \\ d & 0\end{array}\right) \mid a, d \in \mathbb{Z}_{2}\right\}\right)$. Suppose there is a nonzero $R$-homomorphism $f \in \operatorname{Hom}(E(S), S)$, so that $S \cong$ $E(S) / \operatorname{ker}(f)$. Since $S$ is not injective, necessarily $\operatorname{ker}(f) \neq 0$. It follows that $\operatorname{ker}(f)$ is essential in $E(S)$, and thence that $E(S) / \operatorname{ker}(f)$ is a singular module. This is a contradiction because a simple module cannot be both singular and projective. As $S$ embeds in $E(S)$ but it is not one of its quotients, then $S$ is not a parainjective module.

(3) Let $R$ denote Cozzens' domain of differential polynomials, described in [7] (and also in [5, Example B.3]). Those sources provide that $R$ is a left V-ring and a left and right principal ideal domain. As $R$ is a left $\mathrm{V}$-ring, each simple module is injective, so each simple module is parainjective. Note that, as any left principal ideal domain is clearly left hereditary (since, for $0 \neq a \in R, R \cong R a$ ), $R$ is a left and right hereditary ring, and thus any quotient of an injective module is injective. We claim that $R$ is not left injective. Indeed, recall the general notion of 
divisibility: over an arbitrary ring $S$, a module $M$ is divisible if $s M=M$ for each nonzero-divisor $s$. Since $R$ is a domain, every nonzero element of $R$ is a nonzerodivisor. Take any polynomial $g \in R$ of degree greater than 0 . As $1 \notin g R, g R \neq R$, so that ${ }_{R} R$ is not divisible. Therefore, according to [14, Chap. I, $\S 6$, Example 4], ${ }_{R} R$ is not injective. If there was $0 \neq f \in \operatorname{Hom}(E(R), R)$ then $0 \neq \operatorname{Im}(f)$ would be a proper direct summand of $R$, but this is impossible because $R$, being a domain, is directly indecomposable (indeed, for idempotent $e \in R, e(1-e)=0$, so that either $e=0$ or $e=1)$. Hence $\operatorname{Hom}(E(R), R)=0$, thus $R$ is not left mod-retractable.

Definition 2.5. We say that a ring $R$ satisfies condition $(H H)$ if for each two $R$-modules $M, N$

$$
\operatorname{Hom}(M, N) \neq 0 \Leftrightarrow \operatorname{Hom}(N, M) \neq 0
$$

holds.

Example 2.6. Any semisimple ring satisfies $(H H)$.

Recall that a $\operatorname{ring} R$ is said to be left local if there is only one isomorphism class of simple $R$-modules (that is, if every two simple $R$-modules are isomorphic).

Example 2.7. A non-semisimple ring satisfying $(H H)$. By Theorem 2.18 below, an example of a non-semisimple left local and left and right perfect ring suffices to this end. Just let us take the commutative local and artinian ring $R=\mathbb{Z}_{p^{n}}$, where $p$ is a prime number and $n>1$ is a natural number. $R$ is not semisimple because $\operatorname{rad}(R) \neq 0$, writing rad for Jacobson's radical. Note also that the simple ideal for this ring gives us an example of a parainjective module (because of $(H H)$ ) which is not injective. (If it was injective, $R$ would be a V-ring. Equivalently, rad would be the zero preradical, but we know this is not the case.)

Remark 2.8. Let $R$ be a ring satisfying $(H H)$. Then, every simple $R$-module is parainjective and paraprojective, so that $R$ is left max and left semiartinian. Also, $R$ is modretractable.

Recall that a class $\mathbb{T}$ of modules is a torsion class if it is closed under quotients, extensions and direct sums. A class $\mathbb{F}$ of modules is a torsion-free class if it is closed under submodules, direct products and extensions. The pair $(\mathbb{T}, \mathbb{F})$ is called a torsion theory if $\mathbb{T}=\{M \in R$-Mod $\mid \operatorname{Hom}(M, N)=0$ for each $N \in \mathbb{F}\}$ and $\mathbb{F}=\{N \in R$-Mod $\mid$ $\operatorname{Hom}(M, N)=0$ for each $M \in \mathbb{T}\}$ (which implies that $\mathbb{T}$ is a torsion class and $\mathbb{F}$ is a torsion-free class). If $\mathbb{T}$ is an hereditary class, or equivalently, if $\mathbb{F}$ is closed under taking injective hulls, we say that $(\mathbb{T}, \mathbb{F})$ is an hereditary torsion theory. We denote as $R$-TORS the big lattice of torsion theories and the lattice of hereditary torsion theories by $R$-tors.

The following theorem can be consulted in [11, Theorem 3.5].

Theorem 2.9. A ring is left mod-retractable if and only if $R$-TORS $\subseteq R$-tors.

We have the following corollary.

Corollary 2.10. If $R$ is a ring that satisfies (HH) then $R$-TORS $\subseteq R$-tors.

Lemma 2.11. If $R$ is a ring that satisfies (HH) then every torsion-free class $\mathbb{F}$ is closed under quotients.

Proof. Assume that $M \in \mathbb{F}$ and $f: M \rightarrow N$ is an epimorphism. Let $\mathbb{T}$ be the module class such that $(\mathbb{T}, \mathbb{F})$ is a torsion theory. If $N \notin \mathbb{F}$, then $t(N) \neq 0$, where $t(N)$ is the 
largest submodule of $N$ belonging to $\mathbb{T}$. Then we have the following commutative diagram

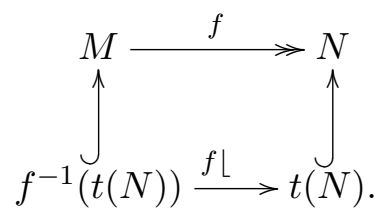

As $f \mid \neq 0$, then by the $(H H)$ condition, there exists a nonzero homomorphism $g \in$ $\operatorname{Hom}\left(t(N), f^{-1}(t(N))\right)$. Thus $g(t(N)) \subseteq t\left(f^{-1}(t(N))\right) \subseteq t(M)=0$, a contradiction. Hence $N \in \mathbb{F}$.

A conatural class is a pseudocomplement in the big lattice $R$-quot of nonempty cohereditary classes in $R$-Mod. $R$-conat denotes the lattice of conatural classes (see [1]).

A module class $\mathcal{C}$ satisfies the condition $(C N)$ if whenever each nonzero quotient of an arbitrary module $M$ shares a nonzero quotient with some element of $\mathcal{C}$, it happens that $M$ belongs to $\mathcal{C}$.

In $[1$, Theorem 23] the following result is proved.

Theorem 2.12. The following conditions are equivalent for a class of modules $\mathfrak{C}$.

(1) $\mathcal{C} \in R$-conat.

(2) $\mathcal{C}$ satisfies $(C N)$.

(3) $\mathcal{C} \in R$-quot and $\mathcal{C}=\left(\mathcal{C}^{\perp_{R-q u o t}}\right)^{\perp_{R-q u o t}}$.

Theorem 2.13. If $R$ is a ring that satisfies $(H H)$, then every torsion class is a conatural class.

Proof. Let $\mathcal{C}$ be a torsion class. Then there is some module class $\mathbb{F}_{\mathcal{C}}$ such that $\left(\mathcal{C}, \mathbb{F}_{\mathcal{C}}\right)$ is a torsion theory. Write $t_{\mathcal{C}}$ for the preradical associated to $\left(\mathcal{C}, \mathbb{F}_{\mathfrak{C}}\right)$. We shall show that $\mathcal{C}$ satisfies condition $(C N)$. Assume that $M \in R$-Mod is such that for each nonzero epimorphism $M \rightarrow N$ there exists a nonzero epimorphism $N \rightarrow K$ with $K \in \mathcal{C}$.

If $M \notin \mathcal{C}$, then $0 \neq M / t_{\mathcal{C}}(M) \in \mathbb{F}_{\mathcal{C}}$. As $M / t_{\mathfrak{C}}(M)$ is a nonzero quotient of $M$, then by hypothesis there exists a nonzero epimorphism $f: M / t_{\mathcal{C}}(M) \rightarrow K$, with $K \in \mathcal{C}$. As $R$ satisfies $(H H)$, there exists $0 \neq g \in \operatorname{Hom}\left(K, M / t_{\mathcal{C}}(M)\right)$, and so $g(K)=g\left(t_{\mathfrak{C}}(K)\right) \subseteq$ $t_{\mathrm{e}}\left(M / t_{\mathrm{e}}(M)\right)=0$, a contradiction. Hence, $M \in \mathrm{e}$.

The following result is part of [3, Proposition 2.11].

Proposition 2.14. The following conditions are equivalent for a ring $R$.

(1) Each simple module is a parainjective module.

(2) Every conatural class is an hereditary torsion class.

Write $\Pi_{1}: R$-TORS $\rightarrow\{\mathbb{T} \mid \mathbb{T}$ is a torsion class of $R$-modules $\}$ for the corresponding projection, which we know is surjective. Thus, $\Pi_{1}(R$-TORS) is the big lattice of torsion classes, and $\Pi_{1}(R$-tors $)$ is the lattice of hereditary torsion classes.

Theorem 2.15. If $R$ is a ring that satisfies $(H H)$, then

$$
\Pi_{1}(R \text {-TORS })=R \text {-conat }=\Pi_{1}(R \text {-tors }) .
$$

Proof. It follows from Theorem 2.13, Remark 2.8 and Proposition 2.14, and from the fact that $R$-tors $\subseteq R$-TORS.

Definition 2.16. We say that a ring $R$ is a $B K N$-ring if $\operatorname{Hom}(M, N) \neq 0$ for each nonzero $R$-modules $M$ and $N$. 
Lemma 2.17. The following conditions are equivalent for a ring $R$.

(1) $R$ is a BKN-ring.

(2) $R$ satisfies $(H H)$ and it is a left local ring.

Proof. $(1) \Rightarrow(2)$ It is clear that $R$ satisfies $(H H)$. As there are nonzero homomorphisms between any two simple modules, all simple modules are isomorphic.

$(2) \Rightarrow(1)$ Let $M$ and $N$ are two nonzero modules. Since by Remark $2.8, R$ is left semiartinian, there is a simple submodule $S$ (resp. $T$ ) of $M$ (resp. $N$ ). As $R$ is left local, $S \cong T$. Because of condition $(H H)$, there is a nonzero homomorphism $f: M \rightarrow S$. The composition $M \stackrel{f}{\rightarrow} S \cong T \hookrightarrow N$ is a nonzero homomorphism from $M$ to $N$.

It is worth noting that, as $R$-nat and $R$-conat are complete lattices of module classes, for an arbitrary module $M$ there is a least member of each of those lattices to which $M$ belongs. We denote as $\xi_{\text {nat }}(M)$ the least natural class having $M$ as an element and as $\xi_{\text {conat }}(M)$ the least conatural class having $M$ as an element. We have the following descriptions of the classes generated by $M$ (see [8] and [13]):

$$
\xi_{\text {nat }}(M)=\{U \in R \text {-Mod : each nonzero submodule of } U
$$

shares a nonzero submodule with $M\}$

and

$$
\begin{aligned}
\xi_{\text {conat }}(M)=\{U \in R \text {-Mod : } & \text { each nonzero quotient of } U \\
& \text { shares a nonzero quotient with } M\} .
\end{aligned}
$$

Theorem 2.18. The following statements are equivalent for a ring $R$.

(1) $R$ satisfies the $(H H)$ condition.

(2) $R$ is mod-retractable and every simple module is paraprojective.

(3) Every simple $R$-module is parainjective and paraprojective.

(4) $R$-nat $=R$-conat.

(5) $R$-nat $=R$-conat $=\Pi_{1}(R$-tors $)$.

(6) $R=R_{1} \times R_{2} \times \cdots \times R_{n}$ where each $R_{i}$ is a BKN-ring.

(7) $R=R_{1} \times R_{2} \times \cdots \times R_{n}$ where each $R_{i}$ is a left local and left and right perfect ring.

(8) $R=R_{1} \times R_{2} \times \cdots \times R_{n}$ where each $R_{i}$ is a full matrix ring over a local left and right perfect ring.

Proof. (1) $\Rightarrow(2)$ It follows from Remark 2.8.

$(2) \Rightarrow(3)$ It follows from Remark 2.3.

$(3) \Rightarrow(1)$ Let $M, N \in R$-Mod and $0 \neq f \in \operatorname{Hom}(M, N)$. By Remark $2.3 R$ is left semiartinian, so there exists some simple module $S \leq \operatorname{Im}(f)$.

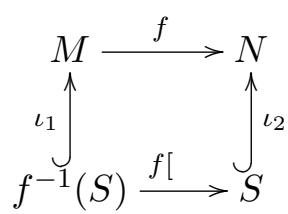

Since $S$ is paraprojective, there exist a monomorphism $g: S \longrightarrow f^{-1}(S)$. On the other hand, $S$ being embedded in $N$ and $S$ being parainjective imply that there exists a epimorphism $h: N \longrightarrow S$. Therefore $\iota_{1} \circ g \circ h: N \longrightarrow M$ is a nonzero homomorphism. $(3) \Leftrightarrow(5) \Leftrightarrow(6) \Leftrightarrow(7) \Leftrightarrow(8)$ They follow from [2, Theorem 4.7].

For $(4) \Rightarrow(3)$ note that if the lattices $R$-nat and $R$-conat coincide, given an arbitrary module $M$ the natural class generated by $M$ is the same class as the conatural class generated by $M$. Thus, if $S$ is a simple submodule of $M$, then $S \in \xi_{\text {nat }}(M)=\xi_{\text {conat }}(M)$. By the description given above, we have that $S$ is a quotient of $M$. Therefore, $S$ is a parainjective module. Symmetrically, if $S$ is a simple quotient of $M$, then $\mathrm{S}$ belongs to 
$\xi_{\text {nat }}(M)$, so that $S$ embeds in $M$. This means that $S$ is a paraprojective module.

$(5) \Rightarrow(4)$ This is clear.

\section{Left duo rings and left exact radicals}

We shall denote as $R$-ler the set of left exact radicals in $R$-Mod, and as $R$-rad the class of all radicals in $R$-Mod. For any two modules $M, N$, set

$$
\rho_{M}(N)=\bigcap\{L \leq N \mid N / L \text { embeds in a product of copies of } M\} .
$$

Clearly, $\rho_{M}(N)$ is the least submodule of $N$ producing a quotient of $N$ embeddable in a product of copies of $M$. We include the following known lemma for the reader's convenience.

Lemma 3.1. For $M \in R$-Mod, $\rho_{M}$ is a preradical.

Proof. We show that if $f: A \longrightarrow B$ then $f\left(\rho_{M}(A)\right) \leq \rho_{M}(B)$, or equivalently, that $\rho(A) \leq f^{-1}\left(\rho_{M}(B)\right)$. We have a commutative diagram

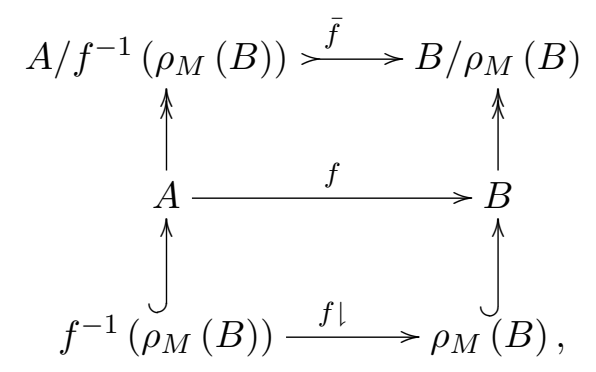

where $\bar{f}$ is a monomorphism. As $B / \rho_{M}(B)$ embeds in a product of copies of $M$, the same occurs for $A / f^{-1}\left(\rho_{M}(B)\right)$. Thus $\rho_{M}(A) \leq f^{-1}\left(\rho_{M}(B)\right)$.

It is clear that $\rho_{M}(N)=0$ if and only if $N$ embeds in a product of copies of $M$. From this it is readily verified that $\rho_{M}$ is a radical. and that it is the greatest preradical $r$ having $M \in \mathbb{F}_{r}$. In particular, each submodule of $M$ belongs to $\mathbb{F}_{\rho_{M}}$.

Remark 3.2. For $Y \in R$-Mod, consider the preradical $\omega_{0}^{Y}$, defined in [9] through

$$
\omega_{0}^{Y}(X)=\bigcap\{\operatorname{ker}(f) \mid f \in \operatorname{Hom}(X, Y)\}
$$

for $X \in R$-Mod. It happens that

$$
\rho_{Y}=\omega_{0}^{Y}
$$

Proof. For each module $X$, both of $\rho_{Y}(X)$ and $\omega_{0}^{Y}(X)$ are the least submodule of $X$ producing a quotient of $X$ cogenerated by $Y$.

(Also, both of $\rho_{Y}$ and $\omega_{0}^{Y}$ are the greatest preradical $r$ such that $r(Y)=0$.)

Lemma 3.3. For an injective module $E, \rho_{E}$ is a left exact radical.

Proof. Write $t_{\chi(E)}$ for the left exact radical corresponding to $\chi(E)$, the hereditary torsion theory cogenerated by $E$ (see [10]). For any module $M$,

$$
\begin{aligned}
M \in \mathbb{F}_{t_{\chi(E)}} & \Longleftrightarrow M \in \mathbb{F}_{\chi(E)} \\
& \Longleftrightarrow M \text { embeds in a product of copies of } E \\
& \Longleftrightarrow M \in \mathbb{F}_{\rho_{E}} .
\end{aligned}
$$

Thus, $\mathbb{F}_{t_{\chi(E)}}=\mathbb{F}_{\rho_{E}}$. But then, $t_{\chi(E)}=\rho_{E}$, as both are radicals. 
(The result can also be obtained using Remark 3.2 and verifying that, whenever $N \leq$ $M \in R$-Mod,

$$
\begin{aligned}
\{\operatorname{ker}(f) \mid f \in \operatorname{Hom}(N, E)\} & \subseteq\{\operatorname{ker}(g \circ \iota) \mid g \in \operatorname{Hom}(M, E)\} \\
& =\{N \cap \operatorname{ker}(g) \mid g \in \operatorname{Hom}(M, E)\},
\end{aligned}
$$

where $N \stackrel{\iota}{\hookrightarrow} M$ is the inclusion map, so that

$$
\begin{aligned}
N \cap \rho_{E}(M) & =N \cap \bigcap\{\operatorname{ker}(g) \mid g \in \operatorname{Hom}(M, E)\} \\
& =\bigcap\{N \cap \operatorname{ker}(g) \mid g \in \operatorname{Hom}(M, E)\} \\
& \leq \bigcap\{\operatorname{ker}(f) \mid f \in \operatorname{Hom}(N, E)\} \\
& \left.=\rho_{E}(N) .\right)
\end{aligned}
$$

Lemma 3.4. Let us consider the following assertions for a ring $R$.

(1) $\rho_{M} \in R$-ler for each $M \in R$-Mod.

(2) $R$-rad $\subseteq R$-ler.

(3) $R$ is a left $V$-ring.

Then $(1) \Leftrightarrow(2) \Rightarrow(3)$ hold. If $R$ is a left semiartinian ring, then $(3) \Rightarrow(1)$.

Proof. (1) $\Rightarrow$ (2) From [9, Proposition 2.1 (2)], and applying Remark 3.2, it follows that for each radical $\rho$,

$$
\rho=\bigwedge_{M \in \mathbb{F}_{\rho}} \rho_{M} .
$$

Thus, (1) implies that each radical is left exact.

(2) $\Rightarrow(1)$ Clear.

$(2) \Rightarrow(3)$ We show that rad is the zero preradical. Let us assume that there exists a module $M$ such that $\operatorname{rad}(M) \neq 0$. Take $0 \neq m \in \operatorname{rad}(M)$. Since rad is left exact, it is idempotent and $\mathbb{T}_{\text {rad }}$ is an hereditary class. We have that $R m \leq \operatorname{rad}(M) \in \mathbb{T}_{\text {rad }}$, so that $R m \in \mathbb{T}_{\text {rad }}$. This means that $\operatorname{rad}(R m)=R m$, that is, $R m$ does not have maximal submodules, a contradiction. Therefore, rad is the zero preradical, which is equivalent to $R$ being a left V-ring.

Now, let us assume that $R$ is a left semiartinian ring.

$(3) \Rightarrow(1)$ By $(3)$ and by Lemma 3.3, $\rho_{S}$ is left exact for every simple module $S$. We shall show that for each $M \in R$-Mod,

$$
\rho_{M}=\bigwedge\left\{\rho_{S} \mid S \in R \text {-simp and } S \text { embeds in } M\right\} .
$$

Let $S$ be a simple module embedding in $M$. As $S$ embeds in $M, \rho_{M}(S)=0$, that is, $S \in \mathbb{F}_{\rho_{M}}$. As $\rho_{S}$ is the largest preradical $r$ such that $S \in \mathbb{F}_{r}, \rho_{M} \leq \rho_{S}$. Hence $\rho_{M} \leq \bigwedge\left\{\rho_{S} \mid S \in R\right.$-simp and $S$ embeds in $\left.M\right\}$. To prove the converse inequality it suffices $(R$-rad being closed under meets) to prove that

$$
\mathbb{F}_{\rho_{M}} \subseteq \mathbb{F} \bigwedge\left\{\rho_{S} \mid S \in R \text {-simp, } \exists S \mapsto M\right\} .
$$

Take $N \in \mathbb{F}_{\rho_{M}}$. Then there is a monomorphism $N \longmapsto M^{X}$ for some set $X$. If

$$
\left(\bigwedge_{\substack{S \in R \text {-simp } \\ \exists S \mapsto M}} \rho_{S}\right)(N) \neq 0,
$$


as $R$ is left semiartinian there exists a simple submodule

$$
T \leq\left(\bigwedge_{\substack{S \in R \text {-simp } \\ \exists S \rightarrow M}} \rho_{S}\right)(N) \leq N
$$

As $N$ embeds in a product of copies of $M, T$ must embed in $M$. But then,

$$
T \leq\left(\bigwedge_{\substack{S \in R \text {-simp } \\ \exists S \succ M}} \rho_{S}\right)(N)=\bigcap_{\substack{S \in R \text {-simp } \\ \exists S \succ M}} \rho_{S}(N) \leq \rho_{T}(N) .
$$

As $\rho_{T}$ is left exact, $\rho_{T}(T)=T \cap \rho_{T}(N)=T$, a contradiction.

Then $\rho_{M}$ is left exact, being the meet of a family of left exact preradicals.

Example 3.5. A non-left-semiartinian ring for which the conditions of the previous lemma are not equivalent.

Let $R$ denote Cozzens' domain mentioned in Example 2.4. As we have noticed, $R$ is a left V-ring and $\operatorname{Hom}(E(R), R)=0$, so that $E(R) \in \mathbb{T}_{\rho_{R}}$. Now, $\rho_{R}$ is not a left exact radical, because otherwise, we would have that $0=\rho_{R}(R)=R \cap \rho_{R}(E(R))=R \cap E(R)=R$, a contradiction. Therefore, we have that $R$ satisfies (3) but does not satisfy (1).

Recall that a ring $R$ is said to be left duo if each one of its left ideals is two-sided. Also, $R$ is strongly regular if for each $x \in R$, there exists $a \in R$ such that $x=a x^{2}$. In what follows, "regular" will mean "regular in the sense of Von Neumann".

The following is part of a theorem which can be consulted in [6].

Theorem 3.6. The following conditions are equivalent for a ring $R$.

(1) $R$ is strongly regular.

(2) $R$ is regular and left duo.

(3) $R$ is left duo and a left $V$-ring.

Lemma 3.7. Let $M$ be a left $R$-module and write $S$ for its endomorphism ring, $\operatorname{End}\left({ }_{R} M\right)$. If $\rho_{M}$ is a left exact radical and $S$ is a regular ring, then $M$ contains an injective nonzero submodule.

Proof. Consult [12, Lemma 4], keeping in mind Remark 3.2.

Theorem 3.8. The following conditions are equivalent for a left duo ring $R$.

(1) $\rho_{M} \in R$-ler, for each left $R$-module $M$.

(2) $R$ is regular and for each left ideal $I$ of $R$ and each left $(R / I)$-module $M, \rho_{M} \in$ $(R / I)$-ler.

(3) $R$ is regular and for each left ideal $I$ of $R, R / I$ contains a nonzero injective left ideal.

(4) $R$ is regular and left semiartinian.

Proof. (1) $\Rightarrow(2)$ By Lemma 3.4 and Theorem 3.6 we have that $R$ is regular. Let $I$ be a left ideal of $R$ and let $M$ be an $(R / I)$-module.

Note that $M$ is also an $R$-module. We shall denote as $\mathbb{F}_{\bar{\rho}_{M}}^{\prime}$ the $\bar{\rho}_{M}$-torsion free $(R / I)$ module class, where $\bar{\rho}_{M}$ is the radical associated to $M$ as an $(R / I)$-module, and as $\mathbb{F}_{\rho_{M}}$ the $\rho_{M}$-torsion free $R$-module class, where $\rho_{M}$ is the radical associated to $M$ as an $R$-module.

Note that $\mathbb{F}_{\bar{\rho}_{M}}^{\prime} \subseteq \mathbb{F}_{\rho_{M}}$. Indeed, take an $(R / I)$-module $N \in \mathbb{F}_{\bar{\rho}_{M}}^{\prime}$. Then $0=\bar{\rho}_{M}(N)=$ $\cap\left\{\operatorname{ker}(f): f \in \operatorname{Hom}_{R / I}(N, M)\right\}$. Now, $\operatorname{Hom}_{R / I}(N, M)=\operatorname{Hom}_{R}(N, M)$ as $N$ and $M$ are both $(R / I)$-modules. Hence, $\rho_{M}(N)=\bar{\rho}_{M}(N)=0$, so that $N \in \mathbb{F}_{\rho_{M}}$.

On the other hand, if $U \in \mathbb{F}_{\rho_{M}}$ is an $R$-module satisfying $I U=0$ then $U$ is an $(R / I)$ module, which gives us that $U \in \mathbb{F}_{\bar{\rho}_{M}}^{\prime}$. 
Since $\bar{\rho}_{M}$ is a radical, in order to prove that it is left exact it will suffice to show that its associated torsion free class, $\mathbb{F}_{\bar{\rho}_{M}}^{\prime}$, is closed under taking injective hulls.

Take $N \in \mathbb{F}_{\bar{\rho}_{M}}^{\prime}$. Let us write $E^{\prime}(N)$ for the injective hull of ${ }_{R / I} N$ and $E(N)$ for the injective hull of $R_{R} N$.

As we have noted above, $N \in \mathbb{F}_{\rho_{M}}$. As $\rho_{M}$ is left exact, it follows that $E(N) \in \mathbb{F}_{\rho_{M}}$.

Over a regular ring, every left or right module is flat. Thus, $(R / I)_{R}$ is flat, and then [12, Lemma 5] provides that ${ }_{R} E^{\prime}(N)$ is injective. It follows that $E^{\prime}(N)=E(N) \in \mathbb{F}_{\rho_{M}}$. Hence, $E^{\prime}(N) \in \mathbb{F}_{\bar{\rho}_{M}}^{\prime}$.

(Alternatively, it is readily verified that $E^{\prime}(N)=\{x \in E(N) \mid I x=0\}$. Thus, we are going to show that $I E(N)=0$ for concluding that $E^{\prime}(N)=E(N)$ as $(R / I)$-modules, from which we will obtain that $E^{\prime}(N) \in \mathbb{F}_{\bar{\rho}_{M}}^{\prime}$.

If $I E(N) \neq 0$, then $N \cap I E(N) \neq 0$. Let us take $0 \neq n \in N \cap I E(N)$. As $(R / I)_{R}$ is flat, from the inclusion $N \stackrel{\iota}{\hookrightarrow} E(N)$ we obtain the monomorphism of $R$-modules

$$
(R / I) \otimes_{R} N \stackrel{1 \otimes \iota}{\longrightarrow}(R / I) \otimes_{R} E(N) .
$$

Then we have, in view of $[14$, Ch. I, $\S 8$, Example 1], that

$$
N \stackrel{\cong}{\longrightarrow} N / I N \stackrel{\cong}{\longrightarrow}(R / I) \otimes_{R} N \stackrel{1 \otimes \iota}{\longrightarrow}(R / I) \otimes_{R} E(N) \stackrel{\cong}{\longrightarrow} E(N) / I E(N) .
$$

This composite sends $n \longmapsto n+I E(N)$, so that $n+I E(N) \neq 0$, contradicting that $n \in \operatorname{IE}(N)$.

Thus, $I E(N)=0$, whence it follows that $E^{\prime}(N)=E(N)$.)

$(2) \Rightarrow(3)$ As $R$ is a regular ring, then each one of its quotient rings is also regular. So, the endomorphism ring $\operatorname{End}(R / I R / I)$ is regular because $\operatorname{End}\left({ }_{R / I} R / I\right)$ is antiisomorphic ${ }^{\ddagger}$ to $R / I$.

By hypothesis, $\rho_{R / I}$ is a left exact radical over $R / I$. Using Lemma 3.7 we have that $R / I$ contains a nonzero injective left ideal.

$(3) \Rightarrow(4)$ By Theorem 3.6, the ring $R$ is strongly regular, being left duo and regular. Then the idempotent elements of $R$ are central (see [14, Ch. I, Lemma 12.2 and Prop. 12.3]). From this point, the proof can continue as 4$) \Rightarrow 5$ ) in [12, Theorem 7].

$(4) \Rightarrow(1)$ As $R$ is left duo and regular, by Theorem 3.6 we have that $R$ is a left Vring. By Lemma 3.4 and since $R$ is left semiartinian, $\rho_{M}$ is left exact radical for each $M \in R$-Mod.

\section{A Galois connection between $R$-nat and $R$-conat}

Definition 4.1. Let $M$ and $N$ be two $R$-modules. We write $M$ Ret $N$ if for each nonzero submodule $M^{\prime} \leq M$ and for each nonzero quotient $N^{\prime}$ of $N, \operatorname{Hom}\left(N^{\prime}, M^{\prime}\right) \neq 0$.

We represent this situation in the diagram

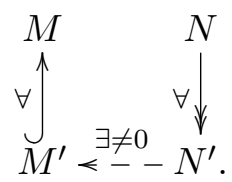

Definition 4.2. We say that a module $M$ is strongly retractable if $M$ Ret $M$, and we say that a ring $R$ is strongly left mod-retractable if each $R$-module is strongly retractable.

Remark 4.3. Note that every strongly retractable module is retractable and that every strongly left mod-retractable ring is left mod-retractable.

†Here, "antiisomorphic to" means "isomorphic to the opposite ring of". 
Example 4.4. Let us take the ring $R=\mathbb{Z}_{p^{n}} \times \mathbb{Z}_{q^{m}}$, where $p$ and $q$ are two different primes and $n, m$ are two natural numbers greater than 1 . By Theorem 2.18(7), we have that $R$ is mod-retractable. On the other hand, $\mathbb{Z}_{p^{n}}$ embeds in $R$ and $\mathbb{Z}_{q^{m}}$ is a quotient of $R$, but $\operatorname{Hom}\left(\mathbb{Z}_{q^{m}}, \mathbb{Z}_{p^{n}}\right)=0$, thus $R$ is not strongly mod-retractable.

We define two classes of modules for each $M, N \in R$-Mod, as follows:

$$
\operatorname{Ret}(M)=\{N \mid M \operatorname{Ret} N\} \text { and } \operatorname{Ret}^{-1}(N)=\{M \mid M \operatorname{Ret} N\} .
$$

Proposition 4.5. For each $M, N \in R$-Mod we have that $\operatorname{Ret}(M)$ is a conatural class and $\operatorname{Ret}^{-1}(N)$ is a natural class.

Proof. We prove that $\operatorname{Ret}(M)$ is a conatural class showing that $\operatorname{Ret}(M)$ satisfies the $(C N)$-condition. Let us suppose that $N \in R$-Mod is such that for each of its nonzero quotients $N^{\prime}$, there exist some nonzero $K \in R$-Mod and some $N^{\prime \prime} \in \operatorname{Ret}(M)$ such that $K$ is a quotient of both $N^{\prime}$ and $N^{\prime \prime}$. As $N^{\prime \prime} \in \operatorname{Ret}(M)$ then $\operatorname{Hom}\left(K, M^{\prime}\right) \neq 0$ for each nonzero submodule $M^{\prime}$ of $M$. It follows that $\operatorname{Hom}\left(N^{\prime}, M^{\prime}\right) \neq 0$. Hence $N \in \operatorname{Ret}(M)$. Therefore, $\operatorname{Ret}(M)$ is a conatural class.

Now, take $M \in \operatorname{Ret}^{-1}(N)$ and let $M^{\prime}$ be a submodule of $M$. If $M^{\prime \prime}$ is a nonzero submodule of $M^{\prime}$, then it is also a submodule of $M$, and thus $\operatorname{Hom}\left(N^{\prime}, M^{\prime \prime}\right) \neq 0$ for each nonzero quotient $N^{\prime}$ of $N$. This proves that $M^{\prime} \in \operatorname{Ret}^{-1}(N)$. Thus $\operatorname{Ret}^{-1}(N)$ is closed under taking submodules.

Now we show that $\operatorname{Ret}^{-1}(N)$ is closed under taking injective hulls. Take a nonzero submodule $H$ of $E(M)$ with $M \in \operatorname{Ret}^{-1}(N)$. As $M \leq_{e} E(M)$, then $H \cap M \neq 0$. Thus for any nonzero quotient $N^{\prime}$ of $N, \operatorname{Hom}\left(N^{\prime}, H \cap M\right) \neq 0$. It follows that $\operatorname{Hom}\left(N^{\prime}, H\right) \neq 0$. To complete the proof, we first show that $\operatorname{Ret}^{-1}(N)$ is closed under extensions. Let us take a short exact sequence with $A, C \in \operatorname{Ret}^{-1}(N)$

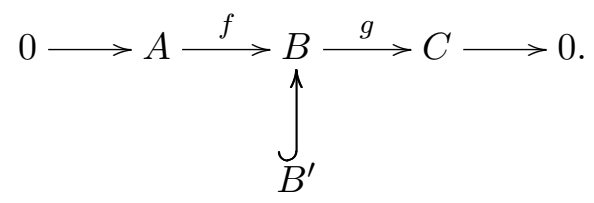

We can assume that $f$ is an inclusion. Let $B^{\prime}$ be a nonzero submodule of $B$. Let us take a nonzero quotient $N^{\prime}$ of $N$. If $B^{\prime} \cap A \neq 0$ then, as $A \in \operatorname{Ret}^{-1}(N)$, there exists a nonzero homomorphism $h: N^{\prime} \longrightarrow B^{\prime} \cap A$, which can be extended to a homomorphism from $N^{\prime}$ to $B^{\prime}$. If $B^{\prime} \cap A=0$, then $B^{\prime}$ embeds in $C$. As $C \in \operatorname{Ret}^{-1}(N)$ then there is a nonzero homomorphism from $N^{\prime}$ to $B^{\prime}$. This shows that $\operatorname{Ret}^{-1}(N)$ is closed under extensions, so in particular, it is closed under taking finite direct sums.

Now, let $\left\{M_{i}\right\}_{i \in I}$ be a family of modules in $\operatorname{Ret}^{-1}(N)$, let $U$ be a nonzero submodule of $\bigoplus_{i \in I} M_{i}$ and let $N^{\prime}$ be a nonzero quotient of $N$. If $0 \neq x \in U$ then $x=m_{i_{1}}+m_{i_{2}}+\cdots+m_{i_{n}}$ with $0 \neq m_{i_{j}} \in M_{i_{j}}$, for $j \in\{i, \ldots, n\}$. As we have already established that the finite direct sum $\bigoplus_{j=1}^{n} M_{i_{j}} \in \operatorname{Ret}^{-1} N$, then there exists a nonzero homomorphism $h: N^{\prime} \longrightarrow R x$, which extends to $U$.

Corollary 4.6. For a class $\mathcal{A}$ of modules, the following statements hold.

$$
\operatorname{Ret}^{-1}(\mathcal{A})=\{M \in R \text {-Mod } \mid M \operatorname{Ret} N \text { for every } N \in \mathcal{A}\} \in R \text {-nat }
$$

and

$$
\operatorname{Ret}(\mathcal{A})=\{N \in R \text {-Mod } \mid M \operatorname{Ret} N \text { for every } M \in \mathcal{A}\} \in R \text {-conat. }
$$

Note that for module classes $\mathcal{A}, \mathcal{B}$, if $\mathcal{A} \subseteq \mathcal{B}$, then $\operatorname{Ret}(\mathcal{B}) \subseteq \operatorname{Ret}(\mathcal{A})$ and $\operatorname{Ret}^{-1}(\mathcal{B}) \subseteq$ $\operatorname{Ret}^{-1}(\mathcal{A})$.

Lemma 4.7. If $\mathcal{A}$ is a class of modules then $\mathcal{A} \subseteq \operatorname{Ret}\left(\operatorname{Ret}^{-1}(\mathcal{A})\right)$ and $\mathcal{A} \subseteq \operatorname{Ret}^{-1}(\operatorname{Ret}(\mathcal{A}))$. 
Proof. It suffices to note that

$$
\operatorname{Ret}^{-1}(\operatorname{Ret}(\mathcal{A}))=\{M \mid M \operatorname{Ret} N \text { for all } N \text { such that } K \operatorname{Ret} N \text { for all } K \in \mathcal{A}\}
$$

and that

$$
\operatorname{Ret}\left(\operatorname{Ret}^{-1}(\mathcal{A})\right)=\{N \mid M \operatorname{Ret} N \text { for all } M \text { such that } M \operatorname{Ret} K \text { for all } K \in \mathcal{A}\}
$$

As a consequence we obtain the following Galois connection (see [14, Ch. III, §8] for the definition).

Theorem 4.8. The following diagram $\S$

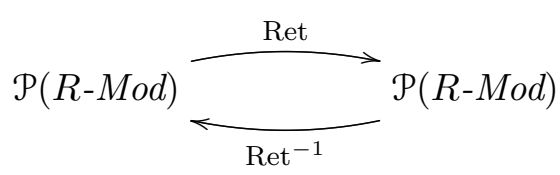

is a Galois connection with closed classes belonging to $R$-conat and to $R$-nat respectively.

Proposition 4.9. For $\mathcal{A}, \mathcal{B} \in R$-nat it happens that

$$
\operatorname{Ret}\left(\mathcal{A} \vee_{\text {nat }} \mathcal{B}\right)=\operatorname{Ret}(\mathcal{A}) \wedge_{\text {conat }} \operatorname{Ret}(\mathcal{B}) \text {. }
$$

Proof. As $\mathcal{A}, \mathcal{B} \subseteq \mathcal{A} \vee_{\text {nat }} \mathcal{B}$, $\operatorname{Ret}\left(\mathcal{A} \vee_{\text {nat }} \mathcal{B}\right) \subseteq \operatorname{Ret}(\mathcal{A}) \wedge_{\text {conat }} \operatorname{Ret}(\mathcal{B})$. For the converse inclusion, it suffices to recall (see [8, Corollary 6.1.7]) that

$$
\mathcal{A} \vee_{\text {nat }} \mathcal{B}=\{M \mid \exists L \leq M \text { such that } L \in \mathcal{A} \text { and } M / L \in \mathcal{B}\}
$$

and to observe that

$\operatorname{Ret}(\mathcal{A}) \wedge_{\text {conat }} \operatorname{Ret}(\mathcal{B})=\left\{N \mid M^{\prime} \operatorname{Ret} N\right.$ and $M^{\prime \prime} \operatorname{Ret} N$ for all $M^{\prime} \in \mathcal{A}$ and for all $\left.M^{\prime \prime} \in \mathcal{B}\right\}$.

Then, use that for each module $X$, the class $\operatorname{Ret}^{-1}(X)$ is natural and thus closed under extensions.

Lemma 4.10. If $M, N \in R$-Mod then

$$
\operatorname{Ret}(M)=\operatorname{Ret}\left(\xi_{\text {nat }}(M)\right) \text { and } \operatorname{Ret}^{-1}(N)=\operatorname{Ret}^{-1}\left(\xi_{\text {conat }}(N)\right) .
$$

Proof. As $\{M\} \subseteq \xi_{\text {nat }}(M)$, we have that $\operatorname{Ret}\left(\xi_{\text {nat }}(M)\right) \subseteq \operatorname{Ret}(M)$. Now take $N \in \operatorname{Ret}(M)$ and $W \in \xi_{\text {nat }}(M)$. We have that for each nonzero submodule $W^{\prime}$ of $W$ there exists a nonzero submodule $W^{\prime \prime}$ of $W^{\prime}$ such that $W^{\prime \prime}$ embeds in $M$. Then $\operatorname{Hom}\left(N^{\prime}, W^{\prime \prime}\right) \neq 0$ for each nonzero quotient $N^{\prime}$ of $N$, which implies $\operatorname{Hom}\left(N^{\prime}, W^{\prime}\right) \neq 0$. Hence $\operatorname{Ret}(M)=$ $\operatorname{Ret}\left(\xi_{\text {nat }}(M)\right)$. The other equality is proved similarly.

Corollary 4.11. If $\mathcal{A}$ is a class of modules then

$$
\operatorname{Ret}(\mathcal{A})=\operatorname{Ret}\left(\xi_{\text {nat }}(\mathcal{A})\right) \text { and } \operatorname{Ret}^{-1}(\mathcal{A})=\operatorname{Ret}^{-1}\left(\xi_{\text {conat }}(\mathcal{A})\right) \text {. }
$$

Recall that any natural class generated by a simple module is an atom in $R$-nat (see [15, Proposition 1.3]).

Proposition 4.12. Let $\mathcal{A} \in R$-nat be an atom generated by a simple module, then $\operatorname{Ret}(\mathcal{A})$ is an atom in $R$-conat.

$\overline{\S_{\text {Let }} \text { us write } \mathcal{P}}(R$-Mod) for the big lattice of classes of $R$-modules, even though $R$-Mod is, of course, not a set. 
Proof. Let $S \in R$-simp be such that $\mathcal{A}=\xi_{\text {nat }}(S)$. Then

$$
\begin{aligned}
\operatorname{Ret}(\mathcal{A}) & =\operatorname{Ret}\left(\xi_{\text {nat }}(S)\right) \\
& =\operatorname{Ret}(S) \\
& =\{N \mid S \operatorname{Ret} N\} \\
& =\left\{N \mid \forall N \rightarrow N^{\prime} \neq 0, \exists N^{\prime} \stackrel{\neq 0}{\longrightarrow} S\right\} \\
& =\xi_{\text {conat }}(S) .
\end{aligned}
$$

Now, any conatural class generated by a simple module is an atom in $R$-conat (see [1, Corollary 40]).

Dually, it can be shown that the image under $\operatorname{Ret}^{-1}$ of atoms generated by simple modules in $R$-conat provides atoms in $R$-nat.

Remark 4.13. The natural class generated by $\mathbb{Z}$ is an atom in $\mathbb{Z}$-nat ( $\mathbb{Z}$ being uniform), but $\operatorname{Ret}(\mathbb{Z})=\{0\}$. (Indeed, take $0 \neq M \in \mathbb{Z}$-Mod. Write $d$ for the divisible part and $t$ for the usual torsion part. If $M$ is divisible, then $M \in \mathbb{T}_{d}$. If $M$ is not divisible, then $n M \lessgtr M$ for some $n>1$, so that $0 \neq M / n M \in \mathbb{T}_{t}$. Either way, seeing as $\mathbb{Z} \in \mathbb{F}_{d} \cap \mathbb{F}_{t}$, it cannot be that $\mathbb{Z}$ Ret $M$.) Thus the image under Ret of an atom does not need to be an atom.

Theorem 4.14. If $R$ is a noetherian ring and $\mathcal{A} \in R$-nat is an atom not generated by a simple module then $\operatorname{Ret}(\mathcal{A})=\{0\}$.

Proof. Assume that there exists a nonzero module $N \in \operatorname{Ret}(\mathcal{A})$. As $\mathcal{A}$ is an atom in $R$ nat, if $M \in \mathcal{A}$ is nonzero, then $\xi_{\text {nat }}(M)=\mathcal{A}$. Thus, $\operatorname{Ret}(\mathcal{A})=\operatorname{Ret}(M)$, so that $M \operatorname{Ret} N$. Hence $\operatorname{Hom}(N, R m) \neq 0$ for each nonzero $m \in M$. As $R m$ is noetherian, $N$ has a nonzero finitely generated quotient. Hence $N$ has a simple quotient $S$. But $S \in \operatorname{Ret}(\mathcal{A})$, because $\operatorname{Ret}(\mathcal{A})$ is a conatural class; thus $M$ Ret $S$. From this we have that $S$ embeds in $M$, thus $\xi_{\text {nat }}(S)=\mathcal{A}$. This constitutes a contradiction.

Theorem 4.15. The following conditions are equivalent for a ring $R$.

(1) $R$ is a left local and left max ring.

(2) $\operatorname{Ret}^{-1}(R-M o d) \neq\{0\}$.

Proof. $(2) \Rightarrow(1)$ Assume that $\operatorname{Ret}^{-1}(R$-Mod $) \neq\{0\}$ and take $0 \neq M \in \operatorname{Ret}^{-1}(R$-Mod). Then $M$ Ret $N$ for each $R$-module $N$. In particular, $M$ Ret $S$ for each $S \in R$-simp. Thus $S$ embeds in each nonzero submodule of $M$. If $S^{\prime}$ is another simple module, then $S^{\prime}$ embeds in $S$, but this is possible only if $S$ and $S^{\prime}$ are isomorphic. Thus all simple modules are isomorphic. As $S \leq M$ and $\operatorname{Ret}^{-1}(R$-Mod $) \in R$-nat, $S \in \operatorname{Ret}^{-1}(R$-Mod). Hence, $S$ Ret $N$ for each $R$-module $N$. Therefore, each nonzero module has a simple quotient.

$(1) \Rightarrow(2)$ If $R$ is a left local and left max ring, then for each $R$-module $N S$ Ret $N$, where $S$ is any simple module. Thus $0 \neq S \in \operatorname{Ret}^{-1}(R$-Mod).

We omit the analogous proof of the following result.

Theorem 4.16. The following conditions are equivalent for a ring $R$.

(1) $R$ is a left local and left semiartinian ring.

(2) $\operatorname{Ret}(R-M o d) \neq\{0\}$.

Proposition 4.17. Let $M, N \in R$-Mod and $S \in R$-simp. If $M \operatorname{Ret} S$ and $S \operatorname{Ret} N$, then $M$ Ret $N$.

Proof. Let $M^{\prime}$ be a nonzero submodule of $M$ and let $N^{\prime}$ be a nonzero quotient of $N$. By hypothesis $\operatorname{Hom}\left(S, M^{\prime}\right) \neq 0$ and $\operatorname{Hom}\left(N^{\prime}, S\right) \neq 0$, whence $\operatorname{Hom}\left(N^{\prime}, M^{\prime}\right) \neq 0$. This means that $M$ Ret $N$. 
Theorem 4.18. The following conditions are equivalent for a ring $R$.

(1) $R$ is a left local and left semiartinian ring.

(2) There exists $S \in R$-simp such that $\operatorname{Ret}^{-1}(S)=R$-Mod.

Proof. (1) $\Rightarrow(2)$ Let $S \in R$-simp. As $R$ is left semiartinian, we have that $\operatorname{Hom}(S, M) \neq 0$ for each nonzero $R$-module $M$. Hence $\operatorname{Ret}^{-1}(S)=R$-Mod.

$(2) \Rightarrow(1)$ If $S \in R$-simp is such that $\operatorname{Ret}^{-1}(S)=R$-Mod, then for each nonzero $R$-module $M$ we have that $M$ Ret $S$ and thus that $\operatorname{Hom}(S, M) \neq 0$. Hence $R$ is left semiartinian. Now, if $S^{\prime} \in R$-simp, as $\operatorname{Hom}\left(S, S^{\prime}\right) \neq 0$, we have that $S$ and $S^{\prime}$ are isomorphic. Thus, $R$ is left local.

Dually, the following theorem is easily proved, and we omit the proof.

Theorem 4.19. The following assertions are equivalent for a ring $R$.

(1) $R$ is a left local, left max ring.

(2) There exists $S \in R$-simp such that $\operatorname{Ret}(S)=R$-Mod.

Corollary 4.20. The following assertions are equivalent for a ring $R$.

(1) $R$ is a left local, left semiartinian and left max ring.

(2) There exists $S \in R$-simp such that $\operatorname{Ret}^{-1}(S)=R-\operatorname{Mod}=\operatorname{Ret}(S)$.

We end this work with a characterization of strongly left mod-retractable rings.

Theorem 4.21. The following assertions are equivalent for a ring $R$.

(1) $R$ is a strongly left mod-retractable ring.

(2) $R$ is a $B K N$-ring.

(3) $R$ satisfies $(H H)$ and it is a left local ring.

(4) $R$ is a left local, left semiartinian and left max ring.

(5) $R$ is left local, left and right perfect ring.

(6) There exists $S \in R$-simp such that $\operatorname{Ret}(S)=R$-Mod $=\operatorname{Ret}^{-1}(S)$.

(7) $R$ is isomorphic to a full matrix ring over a local left and right perfect ring.

Proof. $(1) \Rightarrow(4)$ As $R$ is strongly left mod-retractable, every simple $R$-module is parainjective and paraprojective, so that, by Remark $2.3, R$ is a left semiartinian and left max ring. Now, $R R$ is a strongly retractable module, so that any simple module embeds in any nonzero submodule of ${ }_{R} R$. This implies that there is just one isomorphism type of simple modules.

$(4) \Rightarrow(5)$ As $R$ is left local and left semiartinian, then it is a right perfect ring (see [5, Theorem V.3.4]). Now, every right perfect ring is semilocal, and every semilocal left max ring is left perfect (see, for example, [4, Theorem 28.4]).

$(5) \Rightarrow(4)$ Every left perfect ring is left max, and every right perfect ring is left semiartinian (see, for example, [4, Theorem 28.4]).

$(4) \Rightarrow(2)$ As $R$ is left local, all simple modules are isomorphic. As $R$ is left semiartinian, each simple module embeds in each nonzero module. As the ring is left max, each simple module is a quotient of each nonzero module. If $M, N$ are two nonzero modules, taking an epimorphism from $M$ to a simple module $S$ and composing with a monomorphism from $S$ to $N$, we obtain a nonzero homomorphism from $M$ to $N$.

$(2) \Rightarrow(4)$ Any two simple modules are isomorphic, because for each pair $S, S^{\prime}$ of simple $R$-modules, $\operatorname{Hom}\left(S, S^{\prime}\right) \neq 0$, thus $R$ is left local. As there are nonzero homomorphisms from each simple module to each nonzero module, the ring is left semiartinian. Also, as there are nonzero homomorphisms from each nonzero module to each simple module, then the ring is left max.

$(4) \Leftrightarrow(6)$ This is the preceding corollary.

(6) $\Rightarrow$ (1) It follows from Proposition 4.17. 
$(2) \Leftrightarrow(3)$ This is Lemma 2.17 .

Finally, $(2) \Leftrightarrow(7)$ is a part of [5, Proposition VI.2.3].

Acknowledgment. The authors would like to express their profound gratitude to the referee for the time and attention they devoted to our work. Their pertinent and insightful suggestions were very helpful and greatly improved the contents of this paper.

\section{References}

[1] A. Alvarado García, H. Rincón-Mejía and J. Ríos Montes, On the lattices of natural and conatural classes in R-Mod, Comm. Algebra, 29 (2), 541-556, 2001.

[2] A. Alvarado-García, C. Cejudo-Castilla, H. Rincón-Mejía and I.F. Vilchis-Montalvo, Pseudocomplements and strong pseudocomplements in lattices of module classes, J. Algebra Appl. 17 (1), 1850016, 2018.

[3] A. Alvarado-García, C. Cejudo-Castilla, H. Rincón-Mejía, I.F. Vilchis-Montalvo and M. Zorrilla-Noriega, On boolean lattices of module classes, Algebra Colloq. 25 (2), 285-294, 2018.

[4] F.W. Anderson and K.R. Fuller, Rings and Categories of Modules, Second edition, New York, Springer-Verlag, 1992.

[5] L. Bican, T. Kepka and P. Němec, Rings, Modules and Preradicals, New York, Marcel Dekker, 1982.

[6] K. Chiba and H. Tominaga, On strongly regular rings, Proc. Japan Acad. 49 (6), 435-437, 1973.

[7] J.H. Cozzens, Homological properties of the ring of differential polynomials, Bull. Amer. Math. Soc. 76 (1), 75-79, 1970.

[8] J. Dauns and Y. Zhou, Classes of Modules, Pure and Applied Mathematics, 281, Chapman \& Hall/CRC, Boca Raton FL., 2006.

[9] R. Fernández-Alonso, F. Raggi, J. Ríos, H. Rincón-Mejía and C. Signoret, The lattice structure of preradicals II. Partitions, J. Algebra Appl. 1 (2), 201-214, 2002.

[10] J.S. Golan, Torsion Theories, in:Pitman Monographs and Surveys in Pure and Applied Mathematics, Vol. 29, Longman Scientific and Technical, Harlow, John Wiley and Sons, New York, 1986.

[11] M.T. Koşan and J. Žemlička, Mod-Retractable Rings, Comm. Algebra, 42 (3), 9981010, 2014.

[12] K. Ohtake, Commutative rings of which all radicals are left exact, Comm. Algebra, 8 (16), 1505-1512, 1980.

[13] H. Rincón-Mejía and M. Zorrilla-Noriega, On some relations between the lattices $R$ nat, $R$-conat and $R$-tors and the rings they characterize, J. Algebra Appl. 12 (5), 2013.

[14] B. Stenström, Rings of Quotients, Berlin, Heidelberg, New York, Springer-Verlag, 1975.

[15] Y. Zhou, The lattice of natural classes of modules, Comm. Algebra, 24 (5), 1637-1648, 1996. 